\title{
Importância do conhecimento em dermatologia para atendimento à população ribeirinha do Rio Amazonas
}

\author{
Importance of dermatology knowledge to medical assistance to the riverside population of the \\ Amazon River
}

Rodrigo Santos de Araujo, Elisa M. Amorim da Costa

Como citar esse artigo. Araujo RS, da Costa EMA. Importância do conhecimento em dermatologia para atendimento à população ribeirinha do Rio Amazonas. Revista de Saúde. 2016 Jul./Dez.; $07 \quad$ (2): 04-07.

\begin{abstract}
Resumo
O conhecimento amplo em dermatologia possibilita ao profissional de saúde mais facilidade no diagnóstico e tratamento das afecções que acometem populações que vivem à margem do Rio Amazonas, tendo em vista a incidência de afecções integumentares afligindo estas populações. Este trabalho, um relato de experiência, tem como objetivo documentar a essencial importância do conhecimento dermatológico para profissionais de saúde realizando atendimento a esta população. Este estudo apresenta dados de atendimentos realizados de 08 a 19 de julho de 2013, por um discente da Universidade Severino Sombra, representando a instituição na Operação AssHop (Assistência Hospitalar a populações ribeirinhas do Amazonas), e apresenta uma estatística descritiva dos atendimentos na área de dermatologia. De um total de 176 atendimentos realizados pelo discente sob supervisão médica, 30 (17\%) foram dermatológicos. Destes atendimentos, $33,3 \%$ foram dermatofitoses, $16,6 \%$ corresponderam à pitiríase alba, 16,6 \% foram diagnósticos de pitiríase versicolor e 33,3\% outras dermatopatias, como molusco contagioso, ceratose actínica e dermatite atópica, tendo os pacientes recebido tratamento correspondente. Constatou-se que sem o conhecimento prévio em dermatologia não seria possível atender a $17 \%$ da população necessitando de tratamento médico.
\end{abstract}

Palavras-chave: Dermatologia; População; Rio Amazonas.

\begin{abstract}
Given the incidence of integumentary pathologies affecting riverside populations, such as the one living close to the Amazon River, it is necessary for medical professionals to possess ample knowledge of dermatology in order to easily diagnose conditions affecting patients in these areas. This paper presents data collected during clinical examination of patients during AssHop Operation (Medical Assistance to Riverside Populations of the Amazon River), conducted between the 8th and 19th of July, 2013, during which period a student from Universidade Severino Sombra (Severino Sombra University, USS), always under senior medical staff supervision, conducted 176 clinical examinations. Of all the riverside patients examined, $30(17 \%)$ presented a dermatological condition, with $33.3 \%$ showing dermatophytosis, $16.6 \%$ pityriasis alba, $16.6 \%$ pityriasis versicolor and $33.3 \%$ other pathologies such as molluscum contagiosum, actinic keratosis e atopic dermatitis, with patients being medically treated accordingly. It can be concluded that without previous dermatology knowledge it would not be possible to provide the correct diagnosis and treatment to $17 \%$ of the population requiring medical care.

Keywords: Dermatology; Population; Amazon.
\end{abstract}

\section{Introdução}

Nas últimas décadas a dermatologia apresentou grande crescimento tanto quantitativo como qualitativo. O conhecimento dos mecanismos patogênicos das dermatoses foi muito ampliado, e foram incorporadas novas terapêuticas que têm beneficiado de maneira crescente os portadores de dermatoses. ${ }^{1}$

A Amazônia tem sido objeto de inúmeros estudos que enfocam seuambiente, sociedade e desenvolvimento. Publicações nas últimas décadas discutiram importantes aspectos regionais, como biodiversidade; ciência e tecnologia; crescimento urbano; desenvolvimento sustentável; usos da terra e projetos de desenvolvimento causadores de desmatamento. ${ }^{2,3}$
As dermatofitoses são um exemplo de enfermidade infecciosa com elevada prevalência na América Latina e que atinge tanto o homem como os animais domésticos. ${ }^{4,5}$ Estão entre as zoonoses mais comuns do mundo, sendo consideradas o terceiro distúrbio de pele mais comum em crianças menores de 12 anos e o segundo da população adulta. ${ }^{6}$

Em 2002, o Ministério da Saúde transformou o Guia para o Diagnóstico e Tratamento das Principais Dermatoses da Infância de Interesse Sanitário em Dermatologia na Atenção Básica à Saúde, com intuito de atender uma expressiva demanda dos profissionais da rede básica de saúde, uma vez que as doenças de pele continuam figurando entre as três primeiras causas de demanda aos serviços de saúde. ${ }^{7}$ 
Em um estudo realizado em Manaus-AM entre março e novembro de 2003, pacientes com suspeita clínica de micoses superficiais foram submetidos a exame micológico no período de março a novembro de 2003 no Laboratório de Micologia Médica/CPCS/INPA e verificou-se que de 394 exames, 256 apresentaram diagnóstico positivo para alguma dermatopatia. As micoses mais incidentes foram onicomicoses, Pitiríase versicolor, Malassezia spp e Cândida spp. Tinea capitis apresentou maior ocorrência em pré-escolares e onicomicoses em adultos. O sexo feminino foi o mais acometido. Onicomicoses e Pitiríase versicolor acometeram sobretudo adultos. ${ }^{8}$

As populações que vivem nas comunidades ribeirinhas, que apesar da riqueza e extensão da região, constituem uma população que clama por melhoria de condições de bem-estar econômica e social. ${ }^{9}$ Além disso, deve ser considerada a insuficiência dos meios de transporte e comunicação que estas comunidades enfrentam. Assim, a via fluvial é a mais utilizada forma de transporte nessas localidades. ${ }^{10}$

A atuação de médicos, dentistas e outros agentes de saúde especializados nas comunidades é fato raro e esporádico, principalmente nos locais mais afastados, e este isolamento impõe dificuldades enormes, que fazem com que as populações tradicionais da Amazônia busquem muitas vezes, nas plantas medicinais que cultivam, o tratamento profilático para as doenças. ${ }^{11}$

ASSHOP significa Assistência Hospitalar para as populações ribeirinhas do Amazonas, onde são realizadas operações de assistência médica, odontológica e de orientação sanitária realizadas pela Marinha do Brasil, de forma continuada há mais de 20 anos, com o emprego dos Navios de Assistência Hospitalar pertencentes e tripulados pelo pessoal da Marinha, junto às populações ribeirinhas e indígenas da Amazônia que vivem em comunidades ainda carentes, e na sua grande maioria isoladas da assistência regular de saúde e saneamento básico. ${ }^{12}$

Este estudo objetiva evidenciar as principais patologias dermatológicas que acometem os indivíduos que moram na região amazônica, região essa que recebe médicos formados em outras regiões do Brasil, através do serviço militar. Cada região do país apresenta características epidemiológicas próprias, no que tange a incidência e prevalência de doenças. O conhecimento em dermatologiaé imprescindível para qualquer profissional médico que exerça sua profissão, seja de forma temporária ou definitiva, na Amazônia, visto a grande prevalência de afecções dermatológicas naquela região. Esta pesquisa alerta para o fato de que o profissional de saúde que realizar atendimento na Amazônia precisa estar mais preparado para o atendimento, devendo aprofundar seus conhecimentos em dermatologia, focando nas doenças mais prevalentes.

\section{Materiais e Métodos}

Trata-se de uma pesquisa quantitativa, feita através de levantamento de dados primários. A coleta de dados foi direta e ocasional. Após cada atendimento, foi anotado o sexo do paciente, iniciais do nome e o diagnóstico clínico, já que não havia exames complementares disponíveis. A população total alvo da pesquisa foram 176 pessoas, o que representa o número de atendimentos registrados pelo discente, com amostra de 30 pessoas, utilizando como critério a queixa principal, que deveria possuir caráter dermatológico. Para análise dos dados, foi usado o programa Assistat. Quatro estudantes de medicina, de diferentes faculdades do Brasil (USS, UNIPAM, UEPG e Unifenas) atenderam à população ribeirinha no Navio Hospital Soares de Meirelles nos municípios de Bom Sucesso, Monte Sinai, São José do Amatari, São Pedro de Iracema, Centenário, Urucurituba, Tabocalzinho, Novo Remanso de 08 a 19 de julho. De um total de 637 consultas médicas sob supervisão, 176 foram realizadas pelo discente da Universidade Severino Sombra. A apresentação da distribuição dos dados foi através de percentagem.

\section{Resultados}

A operação AssHop ofereceu atendimento médico a 637 moradores da área ribeirinha do Rio Amazonas, das quais 176 foram realizadas pelo discente de Universidade Severino Sombra, totalizando 28 \% do total. A participação percentual de discentes da cada instituição pode ser vista no gráfico abaixo (Figura 1).

No universo de 176 atendimentos médicos supervisionados realizados pelo discente, representando a Universidade Severino Sombra na operação Asshop,

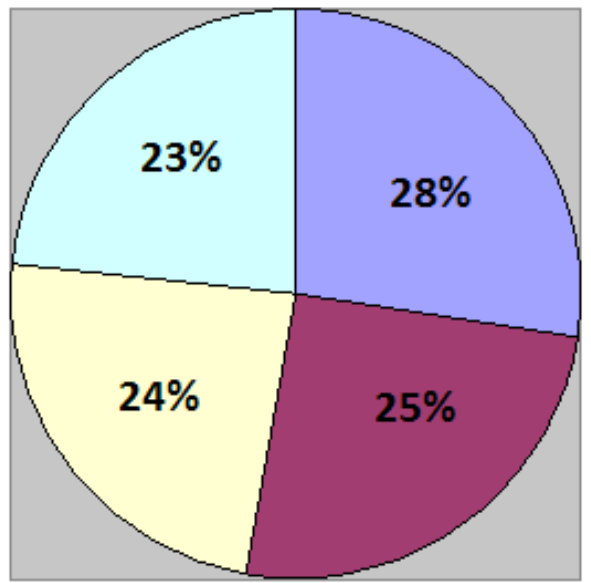

$\square$ Discente USS $\square$ Discente UNIPAM $\square$ Discente Unifenas $\square$ Discente UEPG

Figura 1. Gráfico mostrando o percentual de atendimentos por alunos de cada instituição. Total $=637$ pacientes 
30 pacientes apresentavam como queixa principal lesão dermatológica, correspondendo a $17 \%$ do total. Dos 30 atendimentos dermatológicos, 33,3\% ( $\mathrm{n}=$ 10) dos pacientes foram diagnosticados e tratados como portadores de dermatofitoses; 33,3\% $(n=10)$ receberam diagnóstico de Pitiríase Alba; $16,6 \%(n=5)$ foram diagnósticos de Pitiríase Versicolor e 16,6 \% (n = 5) outras dermatopatias, incluído neste grupo molusco contagioso, ceratose actínica, dermatite atópica, impetigo simples, impetigo bolhoso, furunculose e celulite, sendo iniciado o tratamento específico para cada patologia (Figura 2).

Dos 10 pacientes diagnosticados com dermatofitoses, houve três casos de Tinea pedis; dois casos de Tinea cruris; dois casos de Tinea manus; um caso de Tinea imbricada; um caso de Tinea capitis e um caso de Tine aunguis, também conhecida como onicomicose.

\section{Discussão}

Os resultados evidenciam que queixas dermatológicas têm importância nas consultas em pacientes da região amazônica, correspondendo a 17 $\%$ do total de consultas realizadas pelo discente na operação AssHop. Foram encontrados seis diferentes tipos de dermatofitoses na população atendida. Está em consonância com resultados encontrados pelo autor Oliveira que concluiu que as infecções fúngicas são bastante prevalentes na Amazônia, sendo que em seu estudo as onicomicoses e pitiríase versicolor acometeram principalmente adultos. A Tinea capitis ocorre principalmente em crianças, enquanto que as micoses superficiais apresentaram mais incidentes nas mulheres. ${ }^{8}$

As dermatofitoses corresponderam a $33,3 \%$ das queixas dermatológicas e tem boa resposta ao tratamento com antifúngico tópico, sendo prescrito clotrimazol tópico, antifúngico da classe dos imidazóis, e único disponível no navio Hospital Soares de Meirelles. $\mathrm{O}$ paciente que recebeu o diagnóstico de Tinea capitis, além de receber clotrimazol, recebeu também griseofulvina, visto que a terapêutica oral é essencial dado que os medicamentos tópicos não são capazes de penetrarem adequadamente no folículo e haste capilar. A griseofulvina, disponível desde 1958, é o único antifúngico de administração oral autorizado pela U.S. Food and Drug Administration (FDA) para tratamento das tineas do couro cabeludo nas crianças. ${ }^{13} \mathrm{O}$ paciente com onicomicose recebeu tratamento adicional com fluconazol.

A pitiríase alba (PA) é uma dermatose de etiologia não estabelecida, acometendo principalmente indivíduos atópicos. ${ }^{14}$ Do total de queixas dermatológicas 33,3 $\%$ corresponderam a pitiríase alba e foram orientados quanto a necessidade do uso de protetor solar e a evitar exposição solar entre às 10:00h e 16:00h, não sendo prescrito nenhum tratamento medicamentoso específico.

Ao grupo de pacientes no qual foi diagnosticada pitiríase versicolor foi prescrito clotrimazol tópico, recebendo imediatamente o medicamento na farmácia do navio hospital.

Outras dermatopatias corresponderam a 16,6\% do total de queixas dermatológicas, recebendo tratamento específico para cada patologia. No caso da infecção pelo vírus do molusco contagioso, o paciente foi orientado quanto ao caráter infecto-contagioso da doença, e todas as medidas necessárias para evitar a transmissão para as pessoas com as quais ele convive, além de ser encaminhado ao serviço de dermatologia para que pudesse receber o tratamento específico.

Paciente portador de ceratose actínica recebeu o tratamento com a associação entre diclofenaco e ácido hialurônico tópicos e foram orientados quanto à importância do uso de protetor solar, devido à forte relação da doença com a exposição solar. $\mathrm{O}$ caso de dermatite atópica recebeu hidratante tópico, e foi orientado quanto à necessidade de afastamento de possíveis fatores desencadeantes.

O paciente diagnosticado com impetigo simples e impetigo bolhoso, furunculose e celulite receberam o tratamento com cefalexina $50 \mathrm{mg} / \mathrm{Kg} /$ dia via oral visto

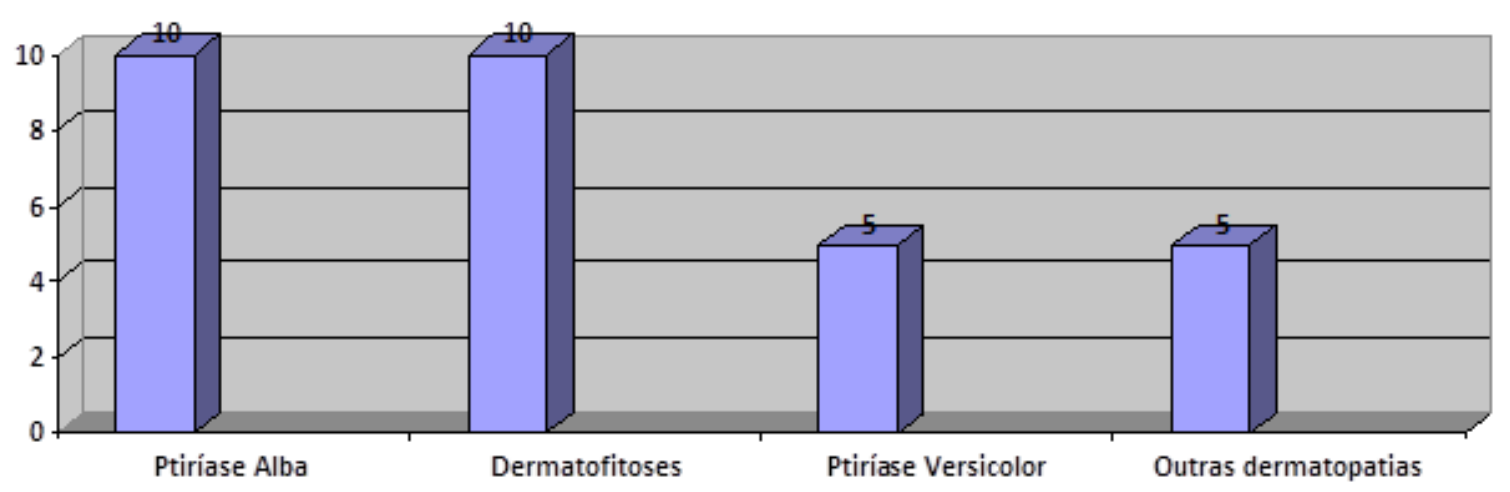

Figura 2. Diagnósticos obtidos a partir de trinta consultas dermatológicas. 
a eficácia dessa droga para as infecções citadas e ser a única droga disponível na farmácia do navio para distribuição para os pacientes.

A Amazônia é uma região quente e úmida, com precariedade de saneamento básico. Os moradores da região ribeirinha têm um contato constante com o Rio Amazonas, áreas de umidade, aliado a isso, possuem condições de higiene precárias. Todos estes fatores contribuem para o alto índice de afecções dermatológicas na população. ${ }^{2}$

A Hanseníase é uma doença prevalente na Amazônia. ${ }^{15} \mathrm{Na}$ operação houve um caso suspeito, já em forma avançada com mutilação dos dedos da mão. Como o critério de seleção de casos da pesquisa foi o fato de a queixa principal do paciente ser dermatológica, este paciente não entrou na contabilização de dados, pois sua queixa principal foi cefaleia. As lesões da hanseníase do paciente citado foram encontradas no exame físico, não gerando queixas no paciente, pois era uma situação que o mesmo afirmava já estar habituado por ser de longa data.

Um fator limitador da pesquisa foi o fato de o diagnóstico ter sido somente clínico, já que não havia nenhum exame complementar disponível. Esse prejuízo não foi tão intenso devido às infecções diagnosticadas terem, na maioria das vezes, o quadro clínico como fator direcionador ao diagnóstico.

\section{Conclusão}

É notória a precariedade de serviços básicos de saúde e saneamento básico da população ribeirinha do Rio Amazonas, que, em sinergismo, contribuem para o alto índice de dermatopatias acometendo a população. Ressalta-se a importância do conhecimento em dermatologia para quaisquer médicos generalistas, sobretudo aqueles que irão trabalhar na região amazônica, visto a prevalência de patologias dermatológicas na região, principalmente infecções fúngicas.

\section{Declaração}

Os autores declaram não haver conflitos de interesse.

\section{Referências}

1. Rivitti EA. Reflexões sobre a dermatologia atual no Brasil. An Bras Dermatol. 2006;81(6):505-7.

2. Faulhaber P, de Toledo PM. Conhecimento e fronteira: história da ciência na Amazônia: Impeg Editoração; 2001.

3. Peres CA. Paving the way to the future of Amazonia. Trends in Ecology \& Evolution. 2001;16(5):217-9.

4. Lacaz CS, Porto E., Martins JEC. Fungos, Actinomicetos, algas e meio ambiente. Epidemiologia das micoses. In: Micologia Médica, Sarvier, São Paulo, 1991, cap. 5, p. 94-106.
5. Rippon J. Dermatophytosis and dermatomycosis. In: Medical mycology. 1988;3:169-275.

6. Murray P, Drew W, Korbay J. Superficial, Cutaneous and Subcutaneous Mycoses. Murray PR, Rosenthal KS, Kobayashi G, Pfaller. In: MA Microbiology Medical. 1994;3:566-76.

7. Ministério da Saúde. Secretaria de Políticas de Saúde Departamento de Atenção Básica Área Técnica de Dermatologia Sanitária. Dermatologia na Atenção Básica de Saúde. Brasilia, 2002.

8. Oliveira JAAD, Barros JDA, Cortez ACA, Oliveira JSRLD. Micoses superficiais na cidade de Manaus, AM, entre março e novembro/2003. An bras dermatol. 2006;81(3):238-43.

9. Schettini AP, Ribas C, Souza IS, Sardinha JCG, Maroja MF, Cunha MG, Picanço N, Cruz RCS. Manual de doenças dermatológicas mais prevalentes. Fundação Alfredo da Mata: Manaus; 2001.

10. Fraxe TJ, Pereira HS, Witkoski AC. Comunidades ribeirinhas amazônicas modos de vida e uso dos recursos naturais: Reggo; 2011.

11. Marinha do Brasil. Disponível em www.mar.mil.br/asshop. Acessado em 01 de agosto de 2014.

12. Hernández T, Machado S, Carvalho S, Selores M. Tinhas do Couro Cabeludo na Idade Pediátrica. Revista Nascer e Crescer. 2004(13 (1)):23-6.

13. Lin RL, Janniger CK. Pityriasis alba. Cutis. 2005;76(1):21-4.

14. Sampaio SA, Castro RM, Rivitti EA. Dermatologia básica. Dermatologia básica: Artes Médicas; 1984 